\title{
ВЫЧИСЛЕНИЕ ЭЛЕМЕНТОВ НАВОДЯЩЕГО ПАКЕТА ПРОГРАММ ДЛЯ ОСОБЫХ КЛАСТЕРОВ МНОЖЕСТВА НАЧАЛЬНЫХ СОСТОЯНИЙ В ЗАДАЧЕ ПАКЕТНОГО НАВЕДЕНИЯ ${ }^{1}$
}

\author{
С. М. Орлов, Н. В. Стрелковский
}

\begin{abstract}
В данной работе рассматривается задача пакетного наведения в заданный момент времени для линейной управляемой динамической системы с конечным множеством начальных состояний. Область управления является выпуклым компактом, а целевое множество - выпуклым и замкнутым. Рассматривается случай наличия особых кластеров в множестве начальных состояний системы, для которых не применим имеющийся алгоритм вычисления элементов наводящего пакета программ. В работе предлагается рассматривать возмущенную задачу расширенного программного наведения со сглаженной областью управления. Доказано, что движения исходной и возмущенной задачи достаточно близки в конечный момент времени, и приводится соответствующая оценка. В случае расширенного целевого множества с непустой внутренностью также показано, что возможно получить решение расширенной задачи программного наведения, точно наводящееся на целевое множество, с помощью применения имеющегося алгоритма для возмущенной задачи со сжатым целевым множеством. Описанная теория иллюстрируется модельным примером.
\end{abstract}

Ключевые слова: неполная информация, линейная динамическая система, задача наведения, пакет программ, особый кластер, гладкая аппроксимация.

S. M. Orlov, N. V. Strelkovskii. Calculation of elements of a guiding program package for singular clusters of the set of initial states in the package guidance problem.

A fixed-time package guidance problem is considered for a linear controlled dynamical system with a finite set of initial states. The control set is convex and compact and the target set is convex and closed. The paper focuses on the case where the set of initial states has singular clusters for which the existing algorithm for estimating the elements of a guiding program package is not applicable. It is suggested to consider a perturbed problem of augmented program guidance with a smoothed control set. It is proved that the motions of the original and perturbed problems are close to each other at the terminal time; the corresponding estimates are provided. In the case of an augmented target set with nonempty interior, it is also shown that a solution of the augmented program guidance problem that is precisely guiding to the target set can be obtained by applying the existing algorithm for the perturbed problem with compressed target set. The suggested theoretical constructions are illustrated with a model example.

Keywords: incomplete information, linear dynamical system, guidance problem, program package, singular cluster, smooth approximation.

MSC: 49M05, 65D10, 93C41

DOI: $10.21538 / 0134-4889-2019-25-1-150-165$

\section{Введение}

Работа посвящена развитию метода пакетов программ [1;2], разработанного Ю. С. Осиповым и А. В. Кряжимским в русле теории позиционного управления [3] и являющегося удобным инструментом для исследования задач гарантирующего управления с неполной информацией, для задачи наведения состояния линейной динамической системы на целевое множество, рассматриваемой в работах [4-9].

Метод основан на утверждении об эквивалентности задач гарантирующего управления в классе позиционных стратегий и в классе пакетов программ. Пакет программ является, по существу, семейством неупреждающих программных "ответов" на все допустимые начальные состояния рассматриваемой динамической системы.

\footnotetext{
${ }^{1}$ Работа выполнена при поддержке РНФ (проект 14-11-00539).
} 
В работах [4-6] для исследования выделяется класс задач, в которых управляемая система и наблюдаемый сигнал линейны, множество допустимых начальных состояний конечно, а целевое множество выпукло и замкнуто. Метод получил естественное обобщение на случай компактного (бесконечного) множества начальных точек использованием конечных аппроксимационных $\varepsilon$-сетей [7]. В работе [7] предложено развитие метода для систем с запаздыванием, в работе [9] рассматривается интегральный наблюдаемый сигнал, а в работе [8] предложено обобщение метода для наведения исследуемой системы на систему множеств. Численный алгоритм нахождения элементов наводящего пакета программ, основанный на методе последовательных приближений, для изначальной постановки задачи предлагается в работе [10]. Однако область его применимости ограничивается случаем, когда все кластеры множества начальных состояний регулярны. В настоящей работе предлагается развитие метода и на случай наличия одного и более особых кластеров множества начальных состояний.

\section{1. Постановка задачи}

\section{1. Задача гарантированного позиционного наведения}

Рассмотрим линейную управляемую динамическую систему вида

$$
\dot{x}(t)=A(t) x(t)+B(t) u(t)+c(t),
$$

где $t$ - время $t \in\left[t_{0}, \vartheta\right], t_{0}<\vartheta<\infty ; x(t) \in \mathbb{R}^{n}-$ состояние системы в момент времени $t$; $u(t) \in \mathbb{R}^{m}$ - значение управления в момент времени $t$. Функции $A(\cdot), B(\cdot), c(\cdot)$ определены и непрерывны на отрезке $\left[t_{0}, \vartheta\right]$ и принимают значения в $\mathbb{R}^{n \times n}, \mathbb{R}^{n \times m}$ и $\mathbb{R}^{n}$ соответственно. Мгновенный ресурс управления $P \subset \mathbb{R}^{m}$ является выпуклым компактом. Произвольную кусочнонепрерывную слева и непрерывную на концах отрезка $\left[t_{0}, \vartheta\right]$ функцию $u(\cdot):\left[t_{0}, \vartheta\right] \mapsto P$ будем называть программой; множество всех программ обозначим через $U$. Для произвольных точки $x_{0} \in \mathbb{R}^{n}$ и программы $u(\cdot) \in U$ решение дифференциального уравнения (1.1) с начальным условием $x\left(t_{0}\right)=x_{0}$, определенное на отрезке $\left[t_{0}, \vartheta\right]$, будем называть движением системы из начального состояния $x_{0}$ под действием программы $u(\cdot)$ и обозначим его как $x\left(\cdot \mid x_{0}, u(\cdot)\right)$.

Управляющей стороне заранее известно, что начальное состояние системы (1.1) содержится в заданном конечном множестве допустимых начальных состояний $X_{0}$, но само это начальное состояние априори не известно. Также заданы выпуклое замкнутое целевое множество $M \subset \mathbb{R}^{n}$ и кусочно-непрерывная слева матрица-функция наблюдения $Q(\cdot)$ на $\left[t_{0}, \vartheta\right]$, принимающая значения в $\mathbb{R}^{q \times n}$ и позволяющая управляющей стороне наблюдать сигнал $y(\cdot): y(t)=Q(t) x(t)$, $t \in\left[t_{0}, \theta\right]$.

Управляющая сторона должна решить задачу гарантирующего позиционного наведения на множество $M$, которая состоит в том, чтобы по заданному $\epsilon>0$ выбрать такую позиционную стратегию управления с памятью (в смысле, определенном в работе [1]), что, каково бы ни было начальное состояние системы $x_{0} \in X_{0}$, движение $x(\cdot)$ системы, соответствующее выбранной позиционной стратегии и исходящее в момент $t_{0}$ из $x_{0}$, в момент $\vartheta$ приходит в состояние $x(\vartheta)$, которое принадлежит $\epsilon$-окрестности $M$.

\section{2. Задача пакетного наведения}

Рассмотрим однородную систему $\dot{x}(t)=A(t) x(t)$, соответствующую $(1.1)$, с начальным условием $x\left(t_{0}\right)=x_{0}$. По формуле Коши ее решение имеет вид $x(t)=F\left(t, t_{0}\right) x_{0}$, где $F(t, s)$, $t, s \in\left[t_{0}, \vartheta\right],-$ матрица Коши. Для каждого допустимого начального состояния $x_{0} \in X_{0}$ определим соответствующий однородный сигнал (функцию) $g_{x_{0}}(\cdot)$ как $g_{x_{0}}(t)=Q(t) F\left(t, t_{0}\right) x_{0}$, $t \in\left[t_{0}, \vartheta\right]$. Отметим, что наблюдение за сигналом исходной системы можно свести к наблюдению за однородным сигналом, воспользовавшись формулой Коши для их связи. Подробнее см. $[6$, п. 6]. Однородный сигнал, соответствующий какому-либо (не специфицированному) 
допустимому начальному состоянию, будем называть просто однородным сигналом и обозначать через $g(\cdot)$. Множество всех допустимых начальных состояний $x_{0} \in X_{0}$, соответствующих однородному сигналу $g(\cdot)$ до момента времени $\tau \in\left[t_{0}, \vartheta\right]$, обозначим через $X_{0}(\tau \mid g(\cdot))$. Таким образом, $X_{0}(\tau \mid g(\cdot))=\left\{x_{0} \in X_{0}:\left.g(\cdot)\right|_{\left[t_{0}, \tau\right]}=\left.g_{x_{0}}(\cdot)\right|_{\left[t_{0}, \tau\right]}\right\}$. Здесь и далее $\left.g(\cdot)\right|_{\left[t_{0}, \tau\right]}$, где $\tau \in\left[t_{0}, \vartheta\right],-$ сужение однородного сигнала $g(\cdot)$ на отрезок $\left[t_{0}, \tau\right]$.

Для произвольного однородного сигнала $g(\cdot)$ определим множество

$$
G_{0}(g(\cdot))=\left\{\tilde{g}(\cdot) \in G: \exists \sigma=\sigma(\tilde{g})>0: \tilde{g}(t) \equiv g(t), t \in\left[t_{0}, t_{0}+\sigma\right]\right\}
$$

исходно совместимых с $g(\cdot)$ однородных сигналов и момент времени

$$
\tau_{1}(g(\cdot))=\max \left\{\tau \in\left[t_{0}, \vartheta\right]: \max _{\tilde{g}(\cdot) \in G_{0}(g(\cdot))} \max _{t \in\left[t_{0}, \tau\right]}|\tilde{g}(t)-g(t)|=0\right\}
$$

который назовем первым моментом расслоения однородного сигнала $g(\cdot)$.

Для $i=1,2, \ldots$ аналогично определим множества

$$
G_{i}(g(\cdot))=\left\{\tilde{g}(\cdot) \in G_{i-1}(g(\cdot)): \tilde{g}(t) \equiv g(t), t \in\left[\tau_{i}(g(\cdot)), \tau_{i}(g(\cdot))+\sigma\right], \sigma=\sigma(\tilde{g})>0\right\}
$$

всех однородных сигналов из $G_{i-1}(g(\cdot))$, совпадающих с $g(\cdot)$ в правосторонней окрестности момента $\tau_{i}(g(\cdot))$, и моменты времени

$$
\tau_{i+1}(g(\cdot))=\max \left\{\tau \in\left(\tau_{i}(g(\cdot)), \vartheta\right]: \max _{\tilde{g}(\cdot) \in G_{i}(g(\cdot))} \max _{t \in\left[\tau_{i}(g(\cdot)), \tau\right]}|\tilde{g}(t)-g(t)|=0\right\},
$$

которые назовем $(i+1)$-ми моментами расслоения однородного сигнала $g(\cdot)$.

Определим также множество $T(g(\cdot))=\left\{\tau_{j}(g(\cdot)): j=1, \ldots, k_{g(\cdot)}\right\}$ всех моментов расслоения однородного сигнала $g(\cdot)$ и множество $T=\cup_{g(\cdot) \in G} T(g(\cdot))$ всех моментов расслоения всех однородных сигналов. Поскольку известно, что множество $T$ конечно [5, п. 2], представим его в виде $T=\left\{\tau_{1}, \ldots, \tau_{K}\right\}$, где $\tau_{1}<\ldots<\tau_{K}$.

Для каждого $k=1, \ldots, K$ определим множество $\mathcal{X}_{0}\left(\tau_{k}\right)=\left\{X_{0}\left(\tau_{k} \mid g(\cdot)\right): g(\cdot) \in G\right\}$ - кластерную позицию в момент $\tau_{k}$, его элементы назовем кластерами начальных состояний в этот момент и будем обозначать через $X_{0 j}\left(\tau_{k}\right), j=1, \ldots, J\left(\tau_{k}\right)$, где $J\left(\tau_{k}\right)$ - количество кластеров в кластерной позиции $\mathcal{X}_{0}\left(\tau_{k}\right), k=1, \ldots, K$.

Семейство программ $\left(u_{x_{0}}(\cdot)\right)_{x_{0} \in X_{0}}$ назовем пакетом программ, если оно удовлетворяет следующему условию неупреждаемости: для любых однородного сигнала $g(\cdot)$, момента $\tau \in\left(t_{0}, \vartheta\right]$ и допустимых начальных состояний $x_{0}^{\prime}, x_{0}^{\prime \prime} \in X_{0}(\tau \mid g(\cdot))$ при всех $t \in\left[t_{0}, \tau\right]$ выполняется равенство $u_{x_{0}^{\prime}}(t)=u_{x_{0}^{\prime \prime}}(t)$. Пакет программ $\left(u_{x_{0}}(\cdot)\right)_{x_{0} \in X_{0}}$ назовем наводящим, если для всякого допустимого начального состояния $x_{0} \in X_{0}$ выполняется включение $x\left(\vartheta \mid x_{0}, u_{x_{0}}(\cdot)\right) \in M$. Если существует какой-либо наводящий пакет программ, то разрешима задача пакетного наведения.

\section{3. Расширенная задача программного наведения}

Для произвольного $h=1,2, \ldots$ введем $\mathcal{R}^{h}-$ конечномерное евклидово пространство всех семейств $\left(r_{x_{0}}\right)_{x_{0} \in X_{0}}$ из $\mathbb{R}^{h}$ со скалярным произведением $\langle\cdot, \cdot\rangle_{\mathcal{R}^{h}}$ вида

$$
\left\langle\left(r_{x_{0}}^{\prime}\right)_{x_{0} \in X_{0}},\left(r_{x_{0}}^{\prime \prime}\right)_{x_{0} \in X_{0}}\right\rangle_{\mathcal{R}^{h}}=\sum_{x_{0} \in X_{0}}\left\langle r_{x_{0}}^{\prime}, r_{x_{0}}^{\prime \prime}\right\rangle_{\mathbb{R}^{h}}, \quad\left(r_{x_{0}}^{\prime}\right)_{x_{0} \in X_{0}},\left(r_{x_{0}}^{\prime \prime}\right)_{x_{0} \in X_{0}} \in \mathcal{R}^{h}
$$

Введем $\mathcal{P} \subset \mathcal{R}^{m}-$ множество всех семейств $\left(u_{x_{0}}\right)_{x_{0} \in X_{0}}$ векторов из множества $P$. Произвольную кусочно-непрерывную слева на отрезке $\left[t_{0}, \vartheta\right]$ функцию $\left(u_{x_{0}}(\cdot)\right)_{x_{0} \in X_{0}}:\left[t_{0}, \vartheta\right] \mapsto \mathcal{P}$ будем называть расширенной программой. Для каждого $k=1, \ldots, K$ обозначим через $\mathcal{P}_{k}$ множество всех семейств векторов $\left(u_{x_{0}}\right)_{x_{0} \in X_{0}} \in \mathcal{P}$ таких, что для всякого кластера $X_{0 j}\left(\tau_{k}\right) \in$ $\mathcal{X}_{0}\left(\tau_{k}\right), j=1, \ldots, J\left(\tau_{k}\right)$, и любых допустимых начальных состояний $x_{0}^{\prime}, x_{0}^{\prime \prime} \in X_{0 j}\left(\tau_{k}\right)$ верно 
$u_{x_{0}^{\prime}}=u_{x_{0}^{\prime \prime}}$ Р Расширенную программу $\left(u_{x_{0}}(\cdot)\right)_{x_{0} \in X_{0}}$ назовем допустимой, если для каждого $k=1, \ldots, K$ выполняются следующие геометрические ограничения: $\left(u_{x_{0}}(t)\right)_{x_{0} \in X_{0}} \in \mathcal{P}_{k}$ при всех $t \in\left(\tau_{k-1}, \tau_{k}\right]$ в случае $k>1$ и при всех $t \in\left[t_{0}, \tau_{1}\right]$ в случае $k=1$. Далее будем обозначать множество допустимых расширенных программ через $\mathcal{U}$, а множество их значений (подмножество множества $\mathcal{P}$, стесненное геометрическими ограничениями, указанными выше) назовем расширенным мгновенным ресурсом управления и обозначим через $\hat{\mathcal{P}}(t), t \in\left[t_{0}, \vartheta\right]$, т. е. $\hat{\mathcal{P}}(t)=\mathcal{P}_{k}$ при всех $t \in\left(\tau_{k-1}, \tau_{k}\right]$ в случае $k>1$ и при всех $t \in\left[t_{0}, \tau_{1}\right]$ в случае $k=1$.

В пространстве $\mathcal{R}^{n}$ рассмотрим динамическую систему, состоящую из экземпляров системы (1.1), параметризованных допустимыми начальными состояниями; движение экземпляра, параметризованного допустимым начальным состоянием $x_{0}$, исходит из него в момент $t_{0}$ и подвергается управлению на отрезке $\left[t_{0}, \vartheta\right]$ некоторой программой $u_{x_{0}}(\cdot)$. Таким образом, расширенная система имеет вид

$$
\left\{\begin{array}{l}
\dot{x}_{x_{0}}(t)=A(t) x_{x_{0}}(t)+B(t) u_{x_{0}}(t)+c(t), \\
x_{x_{0}}\left(t_{0}\right)=x_{0} \in X_{0}
\end{array}\right.
$$

Семейства $\left(u_{x_{0}}(\cdot)\right)_{x_{0} \in X_{0}}$ программ, применяемых для управления расширенной системой, ограничим множеством $\mathcal{U}$. Для каждой допустимой расширенной программы $\left(u_{x_{0}}(\cdot)\right)_{x_{0} \in X_{0}}$ под соответствующим ей движением расширенной системы понимается функция $\left(x\left(\cdot \mid x_{0}, u_{x_{0}}(\cdot)\right)\right)_{x_{0} \in X_{0}}$ : $\left[t_{0}, \vartheta\right] \mapsto \mathcal{R}^{n}$.

Расширенным целевым множеством назовем множество $\mathcal{M}$ всех семейств $\left(x_{x_{0}}\right)_{x_{0} \in X_{0}} \in \mathcal{R}^{n}$ таких, что $x_{x_{0}} \in M$ для всех $x_{0} \in X_{0}$. Допустимая расширенная программа $\left(u_{x_{0}}(\cdot)\right)_{x_{0} \in X_{0}}$ является наводящей для расширенной системы, если выполняется включение

$$
\left(x\left(\vartheta \mid x_{0}, u_{x_{0}}(\cdot)\right)\right)_{x_{0} \in X_{0}} \in \mathcal{M} .
$$

Если существует какая-либо допустимая расширенная программа, являющаяся наводящей для расширенной системы, то разрешима расширенная задача программного наведения.

В работе [5] доказана эквивалентность пакета программ и допустимой расширенной программы в смысле данных выше определений, а также доказана эквивалентность задачи гарантированного позиционного наведения, задачи пакетного наведения и расширенной задачи программного наведения.

\section{2. Вычисление элементов наводящего пакета программ для регулярных кластеров}

Введем следующие обозначения:

$$
\begin{gathered}
D(t)=B^{\mathrm{T}}(t) F^{\mathrm{T}}(\vartheta, t), \quad t \in\left[t_{0}, \vartheta\right], \\
p\left(l, x_{0}\right)=\left\langle l, F\left(\vartheta, t_{0}\right) x_{0}\right\rangle_{\mathbb{R}^{n}}+\left\langle l, \int_{t_{0}}^{\vartheta} F(\vartheta, t) c(t) d t\right\rangle_{\mathbb{R}^{n}}, \quad l \in \mathbb{R}^{n}, \quad x_{0} \in X_{0},
\end{gathered}
$$

а также определим $\rho^{+}(A, l)=\max _{a \in A}\langle a, l\rangle_{E}-$ верхнюю и $\rho^{-}(A, l)=\min _{a \in A}\langle a, l\rangle_{E}, l \in E,-$ нижнюю опорные функции произвольного компактного множества $A$ из евклидова пространства $E$.

Введем функцию $\hat{\gamma}(\cdot, \cdot): \mathcal{R}^{n} \times[0,1] \mapsto \mathbb{R}:$

$$
\begin{gathered}
\hat{\gamma}\left(\left(l_{x_{0}}\right)_{x_{0} \in X_{0}}, a\right)=\sum_{x_{0} \in X_{0}} p\left(l_{x_{0}}, x_{0}\right) \\
+\sum_{k=1}^{K} \int_{\tau_{k-1}}^{\tau_{k}} \sum_{j=1}^{J\left(\tau_{k}\right)} \rho^{-}\left(a P, \sum_{x_{0} \in X_{0 j}\left(\tau_{k}\right)} D(t) l_{x_{0}}\right) d t-\sum_{x_{0} \in X_{0}} \rho^{+}\left(M, l_{x_{0}}\right) .
\end{gathered}
$$


Пусть $\mathcal{L}$ - какое-либо выпуклое компактное множество пространства $\mathcal{R}^{n}$, которое содержит образ единичной сферы $\mathcal{S}^{n}$ при ее, вообще говоря, неравномерном растяжении вдоль всех направлений, т. е. существуют числа $\lambda_{1}$ и $\lambda_{2} \geq \lambda_{1}$ такие, что для каждой точки $z \in \mathcal{S}^{n}$ найдется $\lambda \in\left[\lambda_{1}, \lambda_{2}\right]$, для которого $\lambda z \in \mathcal{L}$.

Здесь и далее будем полагать, что $0 \in \operatorname{int} P$. Расширенную программу $\left(u_{x_{0}}^{0}(\cdot)\right)_{x_{0} \in X_{0}}$ назовем нулевой, если $u_{x_{0}}^{0}(t) \equiv 0, t \in\left[t_{0}, \vartheta\right], x_{0} \in X_{0}$. Известно [6, лемма 3], что если разрешима расширенная задача программного наведения, и при этом нулевая расширенная программа не является наводящей, то существует $a_{*} \in(0,1]$ такое, что

$$
\max _{\left(l_{x_{0}}\right)_{x_{0} \in X_{0}} \in \mathcal{L}} \hat{\gamma}\left(\left(l_{x_{0}}\right)_{x_{0} \in X_{0}}, a_{*}\right)=0
$$

Для произвольной расширенной программы $\left(u_{x_{0}}(\cdot)\right)_{x_{0} \in X_{0}}$, произвольного $k=1, \ldots, K$, произвольного кластера $X_{0 j}\left(\tau_{k}\right) \in \mathcal{X}\left(\tau_{k}\right), j=1, \ldots, J\left(\tau_{k}\right)$, и произвольного момента времени $t \in\left[\tau_{k-1}, \tau_{k}\right)$ обозначим через $u_{X_{0 j}\left(\tau_{k}\right)}(t)$ значения программ $u_{x_{0}}(t)$, одинаковые для всех $x_{0} \in X_{0 j}\left(\tau_{k}\right)$ (с точностью до классов эквивалентности).

Пусть $\left(l_{x_{0}}^{*}\right)_{x_{0} \in X_{0}}$ - какое-либо семейство векторов, максимизирующее выражение $(2.2)$. Назовем кластер $X_{0 j}\left(\tau_{k}\right) \in \mathcal{X}_{0}\left(\tau_{k}\right), k=1, \ldots, K, j=1, \ldots, J\left(\tau_{k}\right)$, регулярным, если

$$
\sum_{x_{0} \in X_{0 j}\left(\tau_{k}\right)} D(t) l_{x_{0}}^{*} \neq 0, \quad t \in\left[\tau_{k-1}, \tau_{k}\right) .
$$

В противном случае будем называть кластер особым.

В следующей теореме сформулировано достаточное условие, аналогичное условию максимума Понтрягина, для наводящей расширенной программы.

Теорема 1 [6, теорема 2]. Пусть множество $P-$ строго выпуклый компакт, $0 \in \operatorname{int} P$, выполняется условие $(2.2)$ и расширенная программа $\left(u_{x_{0}}^{*}(\cdot)\right)_{x_{0} \in X_{0}}$ удовлетворяет условию $u_{x_{0}}^{*}(t) \in a_{*} P, t \in\left[t_{0}, \vartheta\right], x_{0} \in X_{0}$, где $a_{*}-$ корень уравнения $(2.2)$. Пусть кластеры $X_{0 j}\left(\tau_{k}\right) \in$ $\mathcal{X}_{0}\left(\tau_{k}\right), k=1, \ldots, K, j=1, \ldots, J\left(\tau_{k}\right)$, регулярны, и для каждого из них выполняется

$$
\left\langle D(t) \sum_{x_{0} \in X_{0 j}\left(\tau_{k}\right)} l_{x_{0}}^{*}, u_{X_{0 j}\left(\tau_{k}\right)}^{*}(t)\right\rangle=\rho^{-}\left(a_{*} P, D(t) \sum_{x_{0} \in X_{0 j}\left(\tau_{k}\right)} l_{x_{0}}^{*}\right), \quad t \in\left[\tau_{k-1}, \tau_{k}\right) .
$$

Тогда расширенная программа $\left(u_{x_{0}}^{*}(\cdot)\right)_{x_{0} \in X_{0}}$ является наводящей.

На ее основе в работе [10] был предложен алгоритм поиска элементов наводящего пакета программ для регулярных кластеров. Однако в случае наличия особых кластеров хотя бы для одного из моментов расслоения хотя бы на одном из шагов предложенный алгоритм не применим в связи с вырождением условия (2.3). Для иллюстрации этого ограничения рассмотрим следующий пример, отличающийся от примера из работы [10] наличием особого кластера.

П р и м е р 1. Рассмотрим динамическую систему типа "тележка"

$$
\dot{x}_{1}(t)=x_{2}(t), \quad \dot{x}_{2}(t)=u(t)
$$

на отрезке времени $\left[t_{0}, \vartheta\right]=[0,2]$. Управление $u(\cdot)$ принимает значения в множестве $P=[-1,1]$. Множество допустимых начальных состояний $X_{0}$ состоит из двух различных точек

$$
X_{0}=\left\{x_{0}^{\prime}, x_{0}^{\prime \prime}\right\}, \quad \text { где } \quad x_{0}^{\prime}=(-7 / 5,0)^{\mathrm{T}}, \quad x_{0}^{\prime \prime}=(7 / 5,0)^{\mathrm{T}} .
$$

На отрезке времени $[0,1 / 2]$ состояния системы не доступны наблюдению, а на полуотрезке $(1 / 2,2]$ они наблюдаются в полной мере. Таким образом, $Q(\cdot)$ имеет вид

$$
Q(t)= \begin{cases}(0,0), & t \in[0,1 / 2] \\ (1,0), & t \in(1 / 2,2]\end{cases}
$$


Целевое множество имеет вид круга: $M=\left\{\left(x_{1}, x_{2}\right)^{\mathrm{T}} \in \mathbb{R}^{2}: x_{1}^{2}+x_{2}^{2} \leq 1\right\}$. Матрица Коши системы $(2.4)$ и матрица $D(\cdot)$ принимают вид

$$
F(t, s)=\left(\begin{array}{cc}
1 & t-s \\
0 & 1
\end{array}\right), \quad D(t)=\left(\begin{array}{cc}
2-t & 1
\end{array}\right), \quad t, s \in[0,2]
$$

Обозначим $\mathcal{L}=\left(l_{x_{0}^{\prime}}, l_{x_{0}^{\prime \prime}}\right)=\left(\left[l_{x_{0}^{\prime}}^{1}, l_{x_{0}^{\prime}}^{2}\right],\left[l_{x_{0}^{\prime \prime}}^{1}, l_{x_{0}^{\prime \prime}}^{2}\right]\right)$. Максимизируя функцию

$$
\begin{gathered}
\hat{\gamma}(\mathcal{L}, a)=-7 / 5 l_{x_{0}^{\prime}}^{1}+7 / 5 l_{x_{0}^{\prime \prime}}^{1}-a\left(\int_{0}^{1 / 2}\left|(2-t)\left(l_{x_{0}^{\prime}}^{1}+l_{x_{0}^{\prime \prime}}^{1}\right)+\left(l_{x_{0}^{\prime}}^{2}+l_{x_{0}^{\prime \prime}}^{2}\right)\right| d t\right. \\
\left.+\int_{1 / 2}^{2}\left|(2-t) l_{x_{0}^{\prime}}^{1}+l_{x_{0}^{\prime}}^{2}\right| d t+\int_{1 / 2}^{2}\left|(2-t) l_{x_{0}^{\prime \prime}}^{1}+l_{x_{0}^{\prime \prime}}^{2}\right| d t\right)-\sqrt{\left(l_{x_{0}^{\prime}}^{1}\right)^{2}+\left(l_{x_{0}^{\prime}}^{2}\right)^{2}}-\sqrt{\left(l_{x_{0}^{\prime \prime}}^{1}\right)^{2}+\left(l_{x_{0}^{\prime \prime}}^{2}\right)^{2}}
\end{gathered}
$$

по $l=\left(l_{x_{0}^{\prime}}, l_{x_{0}^{\prime \prime}}\right) \in \mathcal{S}^{2}$, где $\mathcal{S}^{2}$ - единичная сфера в пространстве $\mathcal{R}^{2}$, при $a=1$, убедимся, что критерий разрешимости [5] расширенной задачи программного наведения (а следовательно, и соответствующих задач пакетного и гарантирующего позиционного наведения) выполнен. Легко видеть, что кластерные позиции примут следующий вид: $\mathcal{X}_{0}(1 / 2)=\left\{X_{0}\right\}$, а $\mathcal{X}_{0}(2)=$ $\left\{\left\{x_{0}^{\prime}\right\},\left\{x_{0}^{\prime \prime}\right\}\right\}$.

Для вычисления элементов наводящего пакета программ попытаемся применить алгоритм, описанный в работе [10]. На нулевом шаге вычислим значение движения расширенной системы при нулевом расширенном управлении, получим вектор-столбец $d=(-7 / 5,0,7 / 5,0)^{\mathrm{T}}$. Очевидно, что $d \notin \mathcal{M}$, где $\mathcal{M}=M \times M$. Найдем ближайшую к $d$ точку множества $\mathcal{M}[10$, формула (9)]: $z=(-1,0,1,0)^{\mathrm{T}}$. Вычислим нулевое приближение опорного вектора [10, формула (14)]:

$$
l^{*(0)}=\frac{d-z}{\|d-z\|}=\left(-\frac{\sqrt{2}}{2}, 0, \frac{\sqrt{2}}{2}, 0\right)^{\mathrm{T}} .
$$

Поскольку первые два элемента вектора $l^{*(0)}$ соответствуют начальному состоянию $x_{0}^{\prime}$, а остальные элементы - начальному состоянию $x_{0}^{\prime \prime}$, для кластера $X_{0} \in \mathcal{X}_{0}(1 / 2)$ опорный вектор в правой части выражения (2.3) примет вид

$$
\left(\begin{array}{ll}
2-t & 1
\end{array}\right)\left(\begin{array}{c}
-\frac{\sqrt{2}}{2} \\
0
\end{array}\right)+\left(\begin{array}{ll}
2-t & 1
\end{array}\right)\left(\begin{array}{c}
\frac{\sqrt{2}}{2} \\
0
\end{array}\right) \equiv 0, \quad t \in[0,1 / 2),
$$

т. е. кластер $X_{0}$ является особым на полуинтервале $[0,1 / 2)$, и указанный алгоритм далее не может быть применен.

В докладе [11] была сделана попытка обобщить методы, предложенные ранее в работе [12], на рассматриваемую в настоящей работе постановку задачи, однако, несмотря на теоретическую обоснованность предложенных модификаций, их практическая применимость ограничивается чересчур громоздкими вычислениями и низкой скоростью работы алгоритма даже для простейшего примера, аналогичного приведенному. Поэтому в данной работе был предложен альтернативный метод численного поиска решения расширенной задачи программного наведения, заключающийся в аппроксимации множества управления гладким выпуклым компактом и сжатии целевого множества. 


\section{3. Вычисление наводящей расширенной программы в случае наличия особых кластеров}

\section{1. Аппроксимация множества управления гладким выпуклым компактом}

Вернемся к постановке расширенной задачи программного наведения. Для упрощения изложения в соответствии с замечанием 5 работы [5] будем рассматривать задачу не в расширенном пространстве $\mathcal{R}^{n}$, а в евклидовом пространстве $\mathbb{R}^{n N}$, где $N=\left|X_{0}\right|,\left|X_{0}\right|$ - мощность конечного (в силу постановки задачи) множества $X_{0}$, т. е. количество допустимых начальных состояний системы (1.1). Обозначим допустимую расширенную программу, т. е. семейство $u_{x_{0}}(\cdot)_{x_{0} \in X_{0}}$, через $\hat{u}(\cdot)$, соответствующее ей движение расширенной системы $\left(x\left(\cdot \mid x_{0}, u_{x_{0}}(\cdot)\right)\right)_{x_{0} \in X_{0}}$ - через $\hat{x}(\cdot)$, а вектор $\left(x_{0}\right)_{x_{0} \in X_{0}}$ - через $\hat{x}_{0}$. Имеем

$$
\begin{aligned}
& \dot{\hat{x}}(t)=\hat{A}(t) \hat{x}(t)+\hat{B}(t) \hat{u}(t)+\hat{c}(t), \quad \hat{x}\left(t_{0}\right)=\hat{x}_{0}, \\
& \hat{x}(\vartheta) \in \hat{M}, \quad \hat{u}(t) \in \hat{P}(t) .
\end{aligned}
$$

Для каждого $t \in\left[t_{0}, \vartheta\right]$ матрицы $\hat{A}(t) \in \mathbb{R}^{n N \times n N}$ и $\hat{B}(t) \in \mathbb{R}^{n N \times m N}$ являются блочно-диагональными, составленными из $N$ блоков, каждый из которых является матрицей $A(t)$ и $B(t)$ соответственно. Вектор-столбец $\hat{c}(t) \in \mathbb{R}^{n N}$ составляется из записанных друг под другом вектор-столбцов $c(t)$. Множество $\hat{M}$ является декартовым произведением $N$ множеств $M$, а множество $\hat{P}(t)$ - образом множества $\hat{\mathcal{P}}(t) \in \mathcal{R}^{m}$ в пространстве $\mathbb{R}^{m N}$ при соответствующем изометрическом изоморфизме этих пространств, следующем из замечания 5 работы [5].

Выполним замену переменных $v(t)=\hat{B}(t) \hat{u}(t)$, причем

$$
v(t) \in \mathcal{V}(t)=\{v(t)=\hat{B}(t) \hat{u}(t): \hat{u}(t) \in \hat{P}(t)\} .
$$

Тогда задача (3.1) примет вид

$$
\begin{aligned}
& \dot{\hat{x}}(t)=\hat{A}(t) \hat{x}(t)+v(t)+\hat{c}(t), \quad \hat{x}\left(t_{0}\right)=\hat{x}_{0}, \\
& \hat{x}(\vartheta) \in \hat{M}, \quad v(t) \in \mathcal{V}(t) .
\end{aligned}
$$

В работе [10] приведен алгоритм поиска решения расширенной задачи программного наведения в постановке (1.2). Его можно естественным образом перенести на случай постановки задачи (3.2) с произвольным выпуклым компактным множеством $\mathcal{V}(\cdot)$ (это будет означать, что функция (2.1) примет более общий вид). Данный алгоритм применим только в случае отсутствия особых кластеров; это в постановке (3.2) означает, что для каждого направления $l^{i} \in \mathbb{R}^{n N}, i=0, \ldots$, соответствующего $i$-му шагу алгоритма, а также для $l^{*}$, максимизатоpa $(2.2)$, существуют и единственны такие $v^{i}(\cdot), i=1, \ldots$, и $v^{*}(\cdot)$, что

$$
\rho^{-}\left(\mathcal{V}(t), \iota^{i}\right)=\left\langle\complement^{i}, v^{i}(t)\right\rangle, \quad i=1, \ldots, \quad \text { и } \quad \rho^{-}\left(\mathcal{V}(t), \iota^{*}\right)=\left\langle\complement^{*}, v^{*}(t)\right\rangle, \quad t \in[t, \vartheta] .
$$

Фактически возможность применения алгоритма эквивалентна вопросу о том, дает ли условие максимума Понтрягина в задаче (3.2) однозначную информацию об управлении, что и указано в соотношениях (3.3) (в терминах условия минимума). Вообще, условия (3.3) вдоль некоторого вектора $l \in \mathbb{R}^{n N}$ для какого-то $t \in\left[t_{0}, \vartheta\right]$ означают, что в направлении $l$ опорное множество $\mathcal{V}(t)$ состоит из единственной точки $v(t)$. Понятно, что в общем случае это может быть не так, например опорное множество может быть отрезком, гранью, частью гиперплоскости, а если $\mathcal{V}(t)$ имеет пустую внутренность, тогда существует направление $\mathcal{l}$, которое ортогонально всему множеству $\mathcal{V}(t)$, и тогда все множество $\mathcal{V}(t)$ является опорным к вектору $\mathfrak{l}$. В случае задачи пакетного наведения, если информация неполна (задача нетривиальна), то на начальных этапах расслоения однородных сигналов множество $\mathcal{V}(t)$ обязательно будет 
иметь пустую внутренность в силу условий неупреждаемости управлений, каждое из которых понижает размерность множества $\mathcal{V}(t)$. Таким образом, есть ряд задач пакетного наведения, для которых условия (3.3) могут быть не выполнены для всего множества направлений $l^{i} \in \mathbb{R}^{n N}, i=0, \ldots$, и алгоритм из работы [10] к этим задачам напрямую нельзя применить. $\mathrm{B}$ данной ситуации предлагается использовать приближения области управления $\mathcal{V}(\cdot)$ при помощи гладких выпуклых компактов [13], которые являются также строго выпуклыми, что гарантирует выполнение условий (3.3) для любого направления $l \in \mathbb{R}^{n N}$ по определению, и справедлива следующая формула для минимизатора $v(\cdot)$ опорной функции $\rho^{-}(\mathcal{V}(\cdot), \mathcal{l})$ вдоль направления $\mathcal{l}$, которая может быть использована в алгоритме для явного вычисления минимизатора в формуле (2.3):

$$
v(t)=\frac{d}{d \iota} \rho^{-}(\mathcal{V}(t), \mathcal{l}), \quad t \in[t, \vartheta] .
$$

Тогда можно алгоритм из работы [10] применить к возмущенной задаче с гладким выпуклым компактом и строить приближения к решению исходной задачи. Ниже обосновывается соответствующая оценка для приближений, а также предлагается подход к получению приближенного решения исходной задачи, точно наводящегося на целевое множество при помощи дополнительного сжатия целевого множества.

Введем множество $\mathcal{V}_{\varepsilon}(t)$ со следующими свойствами: для любого $t \in\left[t_{0}, \vartheta\right]$ верно, что $\mathcal{V}(t) \subset \mathcal{V}_{\varepsilon}(t)$ и $h\left(\mathcal{V}_{\varepsilon}(t), \mathcal{V}(t)\right) \leq \varepsilon$, где $h(A, B)$ - расстояние по Хаусдорфу [14, определение 5.3] между множествами $A$ и $B$. Рассмотрим задачу

$$
\begin{aligned}
& \dot{\hat{x}}(t)=\hat{A}(t) \hat{x}(t)+v(t)+\hat{c}(t), \quad \hat{x}\left(t_{0}\right)=\hat{x}_{0}, \\
& \hat{x}(\vartheta) \in \hat{M}, \quad v(t) \in \mathcal{V}_{\varepsilon}(t) .
\end{aligned}
$$

Теорема 2. Пусть $\mathcal{V}_{\varepsilon}(t), \varepsilon>0,-$ гладкий выпуклый компакт и пара $\left(\hat{x}_{\varepsilon}(\cdot), v_{\varepsilon}(\cdot)\right)$ является решением задачи (3.4). Тогда для любого управления $v(\cdot): v(t) \in \mathcal{V}(t), t \in[0, \theta]$, такого, что $\left\|v_{\varepsilon}(t)-v(t)\right\| \leq \varepsilon$, соответствующее значение движения $x(\cdot)$ системы (3.2) в момент времени $\theta$ отличается по норме от $\hat{x}_{\varepsilon}(\theta)$ не более, чем на $\varepsilon C$, где $C$ - некоторая положительная постоянная.

Д о к а з а т е л ь с т в о. Пусть управление $v_{\varepsilon}(t) \in \mathcal{V}_{\varepsilon}(t), t \in\left[t_{0}, \vartheta\right]$, решает задачу (3.4). Тогда в силу свойств расстояния по Хаусдорфу существует управление $v(t) \in \mathcal{V}(t), t \in\left[t_{0}, \vartheta\right]$, которое удовлетворяет условию $\left\|v_{\varepsilon}(t)-v(t)\right\| \leq \varepsilon$ (здесь норма $\|\cdot\|$ понимается в смысле евклидовой нормы в пространстве $\left.\mathbb{R}^{n N}\right)$. Оценим близость движений $\hat{x}_{\varepsilon}(\cdot)$ и $\hat{x}(\cdot)$, порожденных управлениями $v_{\varepsilon}(\cdot)$ и $v(\cdot)$ соответственно, в момент времени $\vartheta$. Обозначим через $\|\cdot\|_{F}$ норму Фробениуса в пространстве квадратных матриц конечной размерности, а через $\hat{F}(\cdot, \cdot)$ - фундаментальную матрицу системы линейных дифференциальных уравнений задачи (3.2). Тогда справедливы следующие соотношения:

$$
\begin{aligned}
& \quad\left\|\hat{x}_{\varepsilon}(\vartheta)-\hat{x}(\vartheta)\right\|=\left\|\int_{t_{0}}^{\vartheta} \hat{F}(\vartheta, t) v_{\varepsilon}(t) d t-\int_{t_{0}}^{\vartheta} \hat{F}(\vartheta, t) v(t) d t\right\|=\left\|\int_{t_{0}}^{\vartheta} \hat{F}(\vartheta, t)\left[v_{\varepsilon}(t)-v(t)\right] d t\right\| \\
& \leq \int_{t_{0}}^{\vartheta}\left\|\hat{F}(\vartheta, t)\left[v_{\varepsilon}(t)-v(t)\right]\right\| d t \leq \int_{t_{0}}^{\vartheta}\|\hat{F}(\vartheta, t)\|_{F} \cdot\left\|v_{\varepsilon}(t)-v(t)\right\| d t \leq \varepsilon \int_{t_{0}}^{\vartheta}\|\hat{F}(\vartheta, t)\|_{F} d t=\varepsilon C,
\end{aligned}
$$

где $C=\int_{t_{0}}^{\vartheta}\|\hat{F}(\vartheta, t)\|_{F} d t>0$.

3 а м е ч а н и е 1 . В частности, если значения всех элементов фундаментальной матрицы $\hat{F}(\vartheta, t), t \in\left[t_{0}, \vartheta\right]$, ограничены некоторой константой $\bar{C}>0$, тогда справедлива оценка

$$
\left\|\hat{x}_{\varepsilon}(\vartheta)-\hat{x}(\vartheta)\right\| \leq \varepsilon \int_{t_{0}}^{\vartheta}\|\hat{F}(\vartheta, t)\|_{F} d t \leq \varepsilon \int_{t_{0}}^{\vartheta} \sqrt{\sum_{i, j=1}^{n} \bar{C}^{2}} d t=\varepsilon \bar{C} n\left(\vartheta-t_{0}\right) .
$$


Исходное множество $\mathcal{V}(\cdot)$ может иметь пустую внутренность и быть нестрого выпуклым, что влечет вероятность появления особых кластеров в исходной постановке задачи пакетного наведения (3.2). Выше мы показали, что если использовать вместо него гладкий выпуклый компакт $\mathcal{V}_{\varepsilon}(\cdot), \varepsilon>0$, то, во-первых, в малой окрестности решения новой возмущенной задачи (3.4) всегда лежит траектория исходной задачи (3.2), конец которой близок к концу траектории решения возмущенной задачи (3.4), а, во-вторых, для построения приближенного решения возмущенной задачи (3.4) можно использовать алгоритм из статьи [10], сходящийся к точному решению вследствие отсутствия проблемы особых кластеров.

Таким образом, чтобы найти приближенное решение исходной задачи (3.2), нужно рассмотреть возмущенную задачу (3.4) с достаточно малым $\varepsilon>0$ и найти ее приближенное решение при помощи алгоритма из работы [10]. В данном подходе есть две проблемы, а именно:

1) траектория исходной задачи в окрестности точного решения возмущенной задачи не всегда будет точно наводиться на целевое множество (см. пример ниже);

2) специфика итерационного процесса алгоритма приводит к тому, что на каждом шаге получается новое приближение решения, которое попадает в некоторую уменьшающуюся с количеством итераций окрестность целевого множества, т. е. не происходит точного наведения на целевое множество.

Для преодоления этих проблем и получения решения исходной задачи, точно наводящегося на целевое множество, можно поступить следующим образом: нужно навести объект в возмущенной задаче (3.4) внутрь целевого множества так, чтобы удовлетворить оценке из теоремы 2 и погрешности итерационного процесса алгоритма из работы [10]. Тогда любая траектория в $\varepsilon-$ окрестности (по управлению) полученного приближенного решения возмущенной задачи (3.4) будет точно наводиться на целевое множество в исходной задаче (3.2). Подробнее процедура разъяснена ниже.

\section{2. Процедура сжатия целевого множества}

Зафиксируем некоторое $\delta>0$ и обозначим через $\hat{M}_{\delta}$ произвольное выпуклое замкнутое множество, удовлетворяющее условию $\hat{M}_{\delta}+S_{\delta}(0) \subset \hat{M}$ (здесь $S_{\delta}(0)-$ шар радиуса $\delta$ в пространстве $\mathbb{R}^{n N}$ с центром в начале координат). Рассмотрим следующую задачу наведения:

$$
\begin{aligned}
& \dot{\hat{x}}(t)=\hat{A}(t) \hat{x}(t)+v(t)+\hat{c}(t), \quad \hat{x}\left(t_{0}\right)=\hat{x}_{0}, \\
& \hat{x}(\vartheta) \in \hat{M}_{\delta}, \quad v(t) \in \mathcal{V}_{\varepsilon}(t) .
\end{aligned}
$$

Следующая теорема является основным результатом данной работы.

Теорема 3. Пусть int $\hat{M} \neq \varnothing и$ в задаче (3.2) существует решение, конеи, траектории которого попадает внутрь множества $\hat{M}$. Тогда существуют такие $\delta>0$ и множество $\hat{M}_{\delta}: \hat{M}_{\delta}+S_{\delta}(0) \subset \hat{M}$, что справедливы следующие утвержсдения.

1. Для любого $\varepsilon>0$ задача (3.5) разрешима.

2. Существует такое $\varepsilon>0$, что для любого решения $\left(\hat{x}_{\varepsilon \delta}(\cdot), v_{\varepsilon \delta}(\cdot)\right)$ задачи (3.5) кажсде управление $v(\cdot)$, удовлетворяющее условиям $v(t) \in \mathcal{V}(t) u\left\|v(t)-v_{\varepsilon \delta}(t)\right\| \leq \varepsilon$, наводит оббект задачи (3.2) на иелевое множество $\hat{M}$.

Д о к а з а т е л ь с т в о. Сначала докажем первое утверждение теоремы. Пусть $(\hat{x}(\cdot), v(\cdot))$ - решение задачи $(3.2)$ такое, что $\hat{x}(\vartheta) \in \operatorname{int} \hat{M}$. Тогда существует такое $\delta>0$, что $\hat{x}(\vartheta)+S_{\delta}(0) \subset \operatorname{int} \hat{M}$. Зафиксируем любое выпуклое замкнутое множество $\hat{M}_{\delta}$, такое, что $\hat{M}_{\delta}+S_{\delta}(0) \subset \operatorname{int} \hat{M}$ и $\hat{x}(\vartheta) \in \hat{M}_{\delta}$. Тогда для любого $\varepsilon>0$ задача (3.5) разрешима, так как $v(t) \in \mathcal{V}(t) \subset \mathcal{V}_{\varepsilon}(t), t \in\left[t_{0}, \vartheta\right]$ и $\hat{x}(\vartheta) \in \hat{M}_{\delta}$.

Докажем второе утверждение теоремы. Пусть $\left(\hat{x}_{\varepsilon \delta}(\cdot), v_{\varepsilon \delta}(\cdot)\right)$ - решение задачи (3.5). Тогда в соответствии с теоремой 2 для любого управления $v(\cdot): v(t) \in \mathcal{V}(t),\left\|v(t)-v_{\varepsilon \delta}(t)\right\| \leq \varepsilon$ верно, 
что значение в конечный момент времени соответствующей ему траектория $\hat{x}(\cdot)$ системы $(3.4)$ допускает оценку

$$
\left\|\hat{x}_{\varepsilon \delta}(\vartheta)-\hat{x}(\vartheta)\right\| \leq \varepsilon C
$$

где $C=\int_{t_{0}}^{\vartheta}\|\hat{F}(\vartheta, t)\|_{F} d t>0$. Положим $\varepsilon=\delta / C$. Тогда

$$
\left\|\hat{x}_{\varepsilon \delta}(\vartheta)-\hat{x}(\vartheta)\right\| \leq \delta .
$$

Так как $\hat{x}_{\varepsilon \delta}(\vartheta) \in \hat{M}_{\delta}$, то в силу (3.6) $\hat{x}(\vartheta) \in \hat{M}_{\delta}+S_{\delta}(0) \subset \hat{M}$. Второе утверждение полностью доказано.

Таким образом, если исходная задача (3.2) имеет решение, попадающее внутрь целевого множества $\hat{M}$ (равносильно строгому знаку неравенства в критерии разрешимости из работы [5]), то путем небольшого сжатия целевого множества $\hat{M}$ и аппроксимации множества управления гладким выпуклым компактом с достаточно малым параметром $\varepsilon>0$ можно добиться того, что в достаточно малой окрестности любого решения такой возмущенной задачи (3.5) содержатся точные решения исходной задачи (3.2).

Важным следствием из теоремы 3 является возможность нахождения приближенного решения исходной задачи (3.2), точно наводящегося на целевое множество, при применении алгоритма из работы [10] к возмущенной задаче (3.5).

Следствие. Пусть $\left(\hat{x}_{\varepsilon \delta}^{\Delta}(\cdot), v_{\varepsilon \delta}^{\Delta}(\cdot)\right)$ - приближенное решение задачи (3.5), полученное посредством алгоритма из работы [10], $\Delta>0$ - погрешность попадания на иелевое множество $\hat{M}_{\delta}$, m. е. $\min _{\hat{m} \in \hat{M}_{\delta}}\left\|\hat{x}_{\varepsilon \delta}^{\Delta}(\vartheta)-\hat{m}\right\|=\Delta$. Тогда $\hat{x}_{\varepsilon \delta}^{\Delta}(\vartheta) \in \hat{M}_{\delta}+S_{\Delta}(0)$ u, чтобы n. 2 теоремъ 3 был выполнен для приближенного решения $\left(\hat{x}_{\varepsilon \delta}^{\Delta}(\cdot), v_{\varepsilon \delta}^{\Delta}(\cdot)\right)$, нужно между значениями $\varepsilon, \delta, \Delta$ добиться выполнения соотношения

$$
\varepsilon C \leq \delta+\Delta
$$

3 а м е ч а н и е 2. Добиться выполнения условия (3.7) можно двумя способами: увеличением точности аппроксимации множества $\mathcal{V}(\cdot)$ (уменьшением параметра $\varepsilon$ ) или увеличением числа итераций алгоритма из работы [10] (уменьшением параметра $\Delta$ ).

\section{3. Конструктивные способы построения множеств $\mathcal{V}_{\varepsilon}(\cdot)$ и $\hat{M}_{\delta}$. Сглаживание алгебраической суммы отрезков}

Приведенные выше теоремы и следствие не конкретизируют способы построения множеств $\mathcal{V}_{\varepsilon}(\cdot)$ и $\hat{M}_{\delta}$. Для приложений интересно иметь конструктивные формулы для сжатия целевого множества $\hat{M}$ и для аппроксимации множества управления $\mathcal{V}(\cdot)$ гладким выпуклым компактом.

Могут быть предложены следующие конструктивные методы построения множества $M_{\delta}$ :

1) с помощью сжатия множества относительно центра сжатия (см. [12]);

2) с помощью использования геометрической разности (см. определение в [15]) множества $M_{\delta}$ и шара $S_{\delta}(0)$.

Для построения гладкого выпуклого компакта $\mathcal{V}_{\varepsilon}(\cdot)$ могут быть использованы следующие конструктивные методы [13;16]. Процедура нахождения такого множества в литературе называется сглаживанием множества $\mathcal{V}(\cdot)$, так как изначально множество $\mathcal{V}(\cdot)$ может быть не гладко и с пустой внутренностью. В работе [13] доказана теорема о сглаживании выпуклой оболочки суммы выпуклых множеств на основе аппарата опорных функций. Например, с помощью данной теоремы можно построить множество, которое сглаживает выпуклый многогранник. Но при нарастании количества вершин может усложниться формула для опорной функции, так как предложенную формулу из теоремы необходимо применять итеративно столько раз, сколько имеется последовательных объединений точек многогранника. Поэтому 
для множеств, которые часто встречаются в приложениях, в работах $[13 ; 16 ; 17]$ приведены более удобные формулы сглаживания.

В данной работе для примера используется область управления в форме отрезка, симметричного относительно начала координат, а также алгебраическая сумма двух отрезков. Для подобного рода областей управления в работе [13] используется следующая удобная формула. Пусть множество

$$
U_{0}=I_{1}+\ldots+I_{L}, I_{j}=\left[-b_{j}, b_{j}\right], \quad j=1, \ldots, L,
$$

является алгебраической суммой отрезков в пространстве $\mathbb{R}^{n}\left(b_{j} \in \mathbb{R}^{n}, j=1, \ldots, L\right)$; здесь $L \in \mathbb{N}$ - количество суммируемых отрезков. Тогда верхняя опорная функция сглаженного множества (с параметром $\mu>0)$ имеет вид

$$
\rho^{+}\left(U_{\mu}, l\right)=\sum_{j=1}^{L} \sqrt{\left\langle l, b_{j}\right\rangle^{2}+\mu\left[\|l\|^{2}-\frac{\left\langle l, b_{j}\right\rangle^{2}}{\left\|b_{j}\right\|^{2}}\right]} .
$$

3 а м е ч а н и е 3. В формуле (2.1) и в соответствующих формулах алгоритма из работы [10] используется нижняя опорная функция множества управления. Здесь и ниже для множеств $U_{0}$ и $U_{\mu}$ используется верхняя опорная функция, как это было в работах $[13 ; 16 ; 17]$ (в этих работах есть понятие опорной функции, что эквивалентно определению верхней опорной функции из данной работы). Все утверждения ниже можно сформулировать и доказать в терминах нижней опорной функции в силу соотношения эквивалентности $\rho^{+}(U, l)=\rho^{-}(U,-l)$.

В работах $[13 ; 16]$ не было оценено расстояние по Хаусдорфу $h\left(U_{0}, U_{\mu}\right)$ и не было доказано, что $U_{0} \subset U_{\mu}, \mu>0$. Ниже мы докажем указанное вложение и найдем оценку расстояния по Хаусдорфу между исходным и сглаженным множествами для возможности применения результатов теорем 2 и 3.

Утверждение. Пусть множество $U_{0}$ имеет вид (3.8), а множество $U_{\mu}$ задается опорной функиией (3.9). Тогда, во-первых, для любого $\mu>0$ верно включение $U_{0} \subset U_{\mu}$ и, во-вторых, для любого заданного $\varepsilon>0$ можно выбрать такое $\mu>0$, что $h\left(U_{0}, U_{\mu}\right) \leq \varepsilon$.

Д о к а з а т е л ь с т в о. Сначала докажем, что $U_{0} \subset U_{\mu}, \mu>0$. Для этого докажем, что для любого $l \in \mathbb{R}^{n}$ верно, что $\rho^{+}\left(U_{0}, l\right) \leq \rho^{+}\left(U_{\mu}, l\right)$. Заметим что в формуле (3.9) квадратная скобка неотрицательна в силу неравенства Коши - Буняковского. Тогда

$$
\rho^{+}\left(U_{0}, l\right)=\sum_{j=1}^{L}\left|\left\langle l, b_{j}\right\rangle\right|=\sum_{j=1}^{L} \sqrt{\left\langle l, b_{j}\right\rangle^{2}} \leq \sum_{j=1}^{L} \sqrt{\left\langle l, b_{j}\right\rangle^{2}+\mu\left[\|l\|^{2}-\frac{\left\langle l, b_{j}\right\rangle^{2}}{\left\|b_{j}\right\|^{2}}\right]}=\rho^{+}\left(U_{\mu}, l\right) .
$$

Таким образом, верно, что $U_{0} \subset U_{\mu}, \mu>0$.

Теперь оценим расстояние по Хаусдорфу $h\left(U_{0}, U_{\mu}\right)$. В книге [14] доказано, что расстояние по Хаусдорфу есть максимум модуля разности опорных функций множеств, когда вектор $l$ пробегает единичную сферу в пространстве $\mathbb{R}^{n}$. Воспользуемся этим утверждением для оценки:

$$
\left.h\left(U_{0}, U_{\mu}\right)=\max _{\|l\|=1}\left|\rho^{+}\left(U_{\mu}, l\right)-\rho^{+}\left(U_{0}, l\right)\right|=\max _{\|l\|=1} \sum_{j=1}^{L}\left[\sqrt{\left\langle l, b_{j}\right\rangle^{2}+\mu\left[\|l\|^{2}-\frac{\left\langle l, b_{j}\right\rangle^{2}}{\left\|b_{j}\right\|^{2}}\right.}\right]-\left|\left\langle l, b_{j}\right\rangle\right|\right] .
$$

Сомножитель $\mu$ можно оценить сверху единицей, так как уменьшаемое равно единице (поскольку $l$ перебирается по единичной сфере), а вычитаемое всегда неотрицательно и равно нулю, когда $l$ и $b_{j}$ ортогональны. Получаем, что

$$
h\left(U_{0}, U_{\mu}\right) \leq \max _{\|l\|=1} \sum_{j=1}^{L}\left[\sqrt{\left\langle l, b_{j}\right\rangle^{2}+\mu}-\left|\left\langle l, b_{j}\right\rangle\right|\right]
$$


Рассмотрим функцию $f(x)=\sqrt{x^{2}+\mu}-x$, где $x \geq 0$ и $\mu>0$. Очевидно, что это монотонно убывающая выпуклая функция с максимальным значением $f(0)=\sqrt{\mu}$. С учетом данного рассуждения получаем окончательную оценку:

$$
h\left(U_{0}, U_{\mu}\right) \leq \max _{\|l\|=1} \sum_{j=1}^{L}\left[\sqrt{\left\langle l, b_{j}\right\rangle^{2}+\mu}-\left|\left\langle l, b_{j}\right\rangle\right|\right] \leq \sum_{j=1}^{L} \sqrt{\mu}=\sqrt{\mu} L .
$$

Таким образом, необходимо выбрать такое $\mu$, что $\sqrt{\mu} L \leq \varepsilon$, т. е. $\mu \leq(\varepsilon / L)^{2}$.

Для иллюстрации применения теорем 2,3 и следствия с использованием конструктивных методов, описанных в данном разделе, в разд. 4 разобран модельный пример.

\section{4. Пример}

Вернемся к рассмотрению примера 1 данной работы. Запишем расширенную систему в пространстве $\mathbb{R}^{4}$ в виде (3.1), где

$$
\begin{gathered}
\hat{x}(t)=\left(\begin{array}{c}
x_{x_{0}^{\prime} 1}(t) \\
x_{x_{0}^{\prime} 2}(t) \\
x_{x_{0}^{\prime \prime} 1}(t) \\
x_{x_{0}^{\prime \prime} 2}(t)
\end{array}\right), \quad \hat{u}(t)=\left(\begin{array}{c}
0 \\
u_{x_{0}^{\prime}}(t) \\
0 \\
u_{x_{0}^{\prime \prime}}(t)
\end{array}\right), \\
\hat{A}=\left(\begin{array}{llll}
0 & 0 & 0 & 0 \\
0 & 1 & 0 & 0 \\
0 & 0 & 0 & 0 \\
0 & 0 & 0 & 1
\end{array}\right), \quad \hat{B}=\left(\begin{array}{ll}
0 & 0 \\
1 & 0 \\
0 & 0 \\
0 & 1
\end{array}\right), \quad \hat{x}(0)=\left(\begin{array}{c}
x_{01}^{\prime} \\
x_{02}^{\prime} \\
x_{01}^{\prime \prime} \\
x_{02}^{\prime \prime}
\end{array}\right) ;
\end{gathered}
$$

здесь начальные условия $\hat{x}(0)$ взяты из формулы (2.5). Имеем

$$
\begin{cases}\dot{x}_{x_{0}^{\prime} 1}(t)=x_{x_{0}^{\prime} 2}(t), & x_{x_{0}^{\prime} 1}(0)=x_{01}^{\prime} \\ \dot{x}_{x_{0}^{\prime} 2}(t)=u_{x_{0}^{\prime}}(t), & x_{x_{0}^{\prime} 2}(0)=x_{02}^{\prime} \\ \dot{x}_{x_{0}^{\prime \prime} 1}(t)=x_{x_{0}^{\prime \prime 2}}(t), & x_{x_{0}^{\prime \prime} 1}(0)=x_{01}^{\prime \prime} \\ \dot{x}_{x_{0}^{\prime \prime 2}}(t)=u_{x_{0}^{\prime \prime}}(t), & x_{x_{0}^{\prime \prime} 2}(0)=x_{02}^{\prime \prime}\end{cases}
$$

Расширенный мгновенный ресурс управления примет вид

$$
\hat{P}(t)= \begin{cases}P_{1}, & t \in[0,1 / 2), \\ P_{2}, & t \in[1 / 2,2],\end{cases}
$$

где

$$
\begin{aligned}
& P_{1}=\left\{\left(u_{x_{0}}\right)_{x_{0} \in X_{0}} \in[-1,1] \times[-1,1]: u_{x_{0}^{\prime}}=u_{x_{0}^{\prime \prime}}\right\} \\
& P_{2}=\left\{\left(u_{x_{0}}\right)_{x_{0} \in X_{0}} \in[-1,1] \times[-1,1]\right\} .
\end{aligned}
$$

Сделаем замену переменной

$$
v(t)=\hat{B} \hat{u}(t)=\left(\begin{array}{c}
v_{x_{0}^{\prime} 1}(t) \\
v_{x_{0}^{\prime} 2}(t) \\
v_{x_{0}^{\prime \prime} 1}(t) \\
v_{x_{0}^{\prime \prime 2}}(t)
\end{array}\right) \in \mathcal{V}(t)=\hat{B} \hat{P}(t)= \begin{cases}V_{1}, & t \in[0,1 / 2), \\
V_{2}, & t \in[1 / 2,2],\end{cases}
$$

где

$$
\begin{aligned}
& V_{1}=\left\{v \in \mathbb{R}^{4}: v_{x_{0}^{\prime} 1}=v_{x_{0}^{\prime \prime} 1}=0, v_{x_{0}^{\prime} 2}=v_{x_{0}^{\prime \prime 2}} \in[-1,1]\right\} \\
& V_{2}=\left\{v \in \mathbb{R}^{4}: v_{x_{0}^{\prime} 1}=v_{x_{0}^{\prime \prime} 1}=0, v_{x_{0}^{\prime} 2}, v_{x_{0}^{\prime \prime 2}} \in[-1,1]\right\} .
\end{aligned}
$$


Система (4.1) примет вид

$$
\begin{cases}\dot{x}_{x_{0}^{\prime} 1}(t)=x_{x_{0}^{\prime} 2}(t)+v_{x_{0}^{\prime} 1}(t), & x_{x_{0}^{\prime} 1}(0)=x_{01}^{\prime}, \\ \dot{x}_{x_{0}^{\prime} 2}(t)=v_{x_{0}^{\prime} 2}(t), & x_{x_{0}^{\prime} 2}(0)=x_{02}^{\prime}, \\ \dot{x}_{x_{0}^{\prime \prime} 1}(t)=x_{x_{0}^{\prime \prime 2}}(t)+v_{x_{0}^{\prime \prime} 1}(t), & x_{x_{0}^{\prime \prime} 1}(0)=x_{01}^{\prime \prime} \\ \dot{x}_{x_{0}^{\prime \prime 2}}(t)=v_{x_{0}^{\prime \prime 2}}(t), & x_{x_{0}^{\prime \prime 2}}(0)=x_{02}^{\prime \prime}\end{cases}
$$

Заметим, что множества $V_{1}$ и $V_{2}$ имеют вид (3.8) с соответствующими постоянными $L_{V_{1}}=1$, $b_{1}=(0,1,0,1)^{\mathrm{T}}$ - для множества $V_{1}$ и $L_{V_{2}}=2, b_{1}=(0,1,0,0)^{\mathrm{T}}, b_{2}=(0,0,0,1)^{\mathrm{T}}$ - для множества $V_{2}$. Таким образом, множества (4.2), (4.3) можно сгладить при помощи формулы (3.9). Для наглядности возьмем параметр сглаживания $\mu=0.01$.

Теперь применим алгоритм из работы [10] к системе (4.4) со сглаженной областью управления, заданной опорной функцией (3.9) с указанными выше параметрами. После 14 итераций алгоритма погрешность наведения (т. е. расстояние от конца движения системы до целевого множества) составила $\Delta \approx 0.001$. Получим траектории $\left(x_{x_{0}^{\prime}}^{\mu}(\cdot), x_{x_{0}^{\prime \prime}}^{\mu}(\cdot)\right)$ и соответствующие им управления $\left(v_{x_{0}^{\prime}}^{\mu}(\cdot), v_{x_{0}^{\prime \prime}}^{\mu}(\cdot)\right)$. Применяя результаты теоремы 2 , найдем в $\mu$-окрестности полученных управлений управления исходной системы (4.4), а именно возьмем следующие управления:

$$
\begin{array}{llrl}
\bar{v}_{x_{0}^{\prime} 1}(t)=\bar{v}_{x_{0}^{\prime \prime} 1}(t) \equiv 0, & & t \in[0,2], \\
\bar{v}_{x_{0}^{\prime} 2}(t)=\bar{v}_{x_{0}^{\prime \prime 2}}(t)=v_{x_{0}^{\prime} 2}^{\mu}(t), & & t \in[0,1 / 2], \\
\bar{v}_{x_{0}^{\prime} 2}(t)=v_{x_{0}^{\prime} 2}^{\mu}(t), & & t \in[1 / 2,2], \\
\bar{v}_{x_{0}^{\prime \prime 2} 2}(t)=v_{x_{0}^{\prime \prime 2}}^{\mu}(t), & & t \in[1 / 2,2] .
\end{array}
$$

Вообще говоря, можно выбирать различные управления $\left(\bar{v}_{x_{0}^{\prime}}(\cdot), \bar{v}_{x_{0}^{\prime \prime}}(\cdot)\right)$ из $\mu$-окрестности управлений $\left(v_{x_{0}^{\prime}}^{\mu}(\cdot), v_{x_{0}^{\prime \prime}}^{\mu}(\cdot)\right)$; при некоторых управлениях возможно точное попадание на целевое множество, при некоторых - нет. Здесь для иллюстрации выбраны такие управления $\left(\bar{v}_{x_{0}^{\prime}}(\cdot), \bar{v}_{x_{0}^{\prime \prime}}(\cdot)\right)$, что не происходит точного наведения на целевое множество. На рис. 1 изображены траектории $\left(x_{x_{0}^{\prime}}^{\mu}(\cdot), x_{x_{0}^{\prime \prime}}^{\mu}(\cdot)\right)$, соответствующие найденным из алгоритма управлениям $\left(v_{x_{0}^{\prime}}^{\mu}(\cdot), v_{x_{0}^{\prime \prime}}^{\mu}(\cdot)\right)$, а также траектории $\left(\bar{x}_{x_{0}^{\prime}}(\cdot), \bar{x}_{x_{0}^{\prime \prime}}(\cdot)\right)$, соответствующие управлениям $(4.5)$, в пространстве переменных $x_{1}, x_{2}$ (координат движений системы). Видно, что найденные траектории $\left(\bar{x}_{x_{0}^{\prime}}(\cdot), \bar{x}_{x_{0}^{\prime \prime}}(\cdot)\right)$ не наводятся точно на целевое множество, а погрешность наведения не превосходит $\sqrt{\mu} L_{V_{1}} C_{1}+\sqrt{\mu} L_{V_{2}} C_{2}+\Delta$, где

$$
C_{1}=\int_{0}^{1 / 2}\|\hat{F}(2, t)\|_{F} d t \leq 1.6, \quad C_{2}=\int_{1 / 2}^{2}\|\hat{F}(2, t)\|_{F} d t \leq 3.5
$$

в соответствии с результатами утверждения и теорем 2 и 3.

Чтобы точно навести траектории исходной системы (4.4), можно применить результаты теоремы 3 для точного наведения. Применим алгоритм из работы [10] к расширенной задаче программного наведения для системы (4.4) при коэффициенте сглаживания множества управления $\mu=0.0001$ на сжатое целевое множество $\hat{M}_{\delta}=M_{\delta} \times M_{\delta}$, где $M_{\delta}=\left\{\left(x_{0}^{\prime}, x_{0}^{\prime \prime}\right)^{\mathrm{T}}\right.$ : $\left.\left(x_{0}^{\prime}\right)^{2}+\left(x_{0}^{\prime \prime}\right)^{2} \leq(1-\delta)^{2}\right\}, \delta=0.1$. После 17 итераций алгоритма погрешность вычисления составила $\Delta \approx 0.001$. Мы получили траектории $\left(x_{x_{0}^{\prime}}^{\mu \delta}(\cdot), x_{x_{0}^{\prime \prime}}^{\mu \delta}(\cdot)\right)$ и соответствующие им управления $\left(v_{x_{0}^{\prime}}^{\mu \delta}(\cdot), v_{x_{0}^{\prime \prime}}^{\mu \delta}(\cdot)\right)$. Отметим, что выполнено неравенство для погрешностей из следствия теоремы 3

$$
\sqrt{\mu} L_{V_{1}} C_{1}+\sqrt{\mu} L_{V_{2}} C_{2} \leq 0.01 \cdot 1 \cdot 1.6+0.01 \cdot 2 \cdot 3.5=0.086<\delta+\Delta \approx 0.101 .
$$




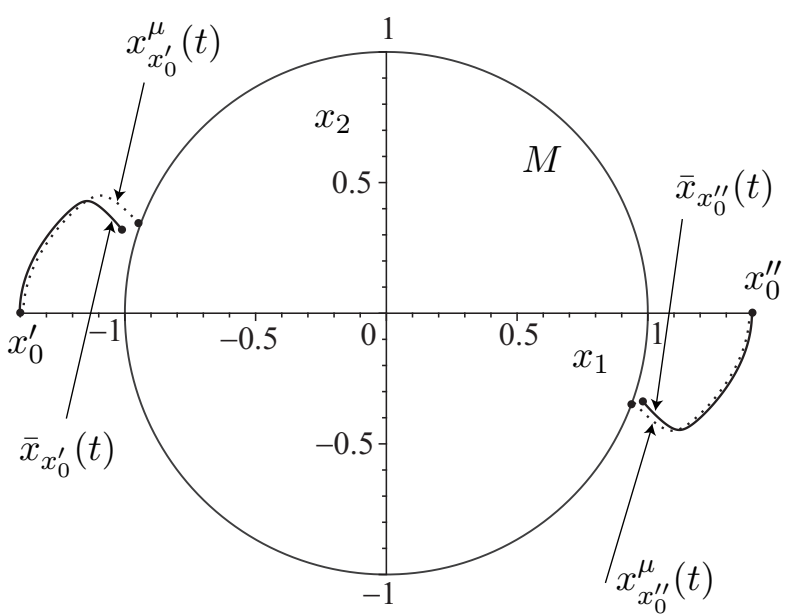

Рис. 1. Наведение на множество $\hat{M}$ (проекция на $\left.\mathbb{R}^{2}\right)$

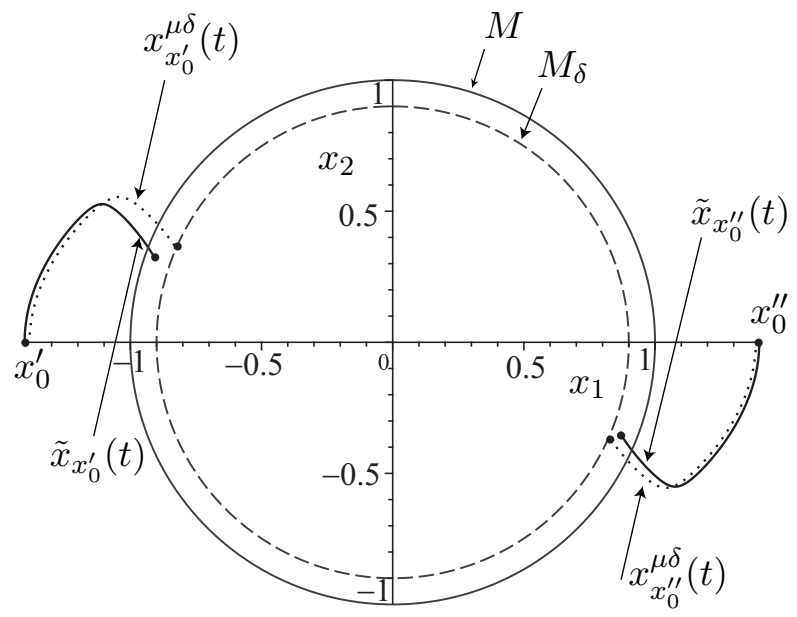

Рис. 2. Наведение на множество $\hat{M}_{\delta}, \delta=0.1$ (проекция на $\mathbb{R}^{2}$ )

Таким образом, траектории, соответствующие любым управлениям из $\mu$-окрестности $\left(v_{x_{0}^{\prime}}^{\mu \delta}(\cdot), v_{x_{0}^{\prime \prime}}^{\mu \delta}(\cdot)\right)$, наводятся точно на целевое множество. В качестве примера были взяты следующие управления:

$$
\begin{array}{lll}
\tilde{v}_{x_{0}^{\prime} 1}(t)=\tilde{v}_{x_{0}^{\prime \prime} 1}(t) \equiv 0, & & t \in[0,2], \\
\tilde{v}_{x_{0}^{\prime} 2}(t)=\tilde{v}_{x_{0}^{\prime \prime 2}}(t)=v_{x_{0}^{\prime} 2}^{\mu \delta}(t), & & t \in[0,1 / 2], \\
\tilde{v}_{x_{0}^{\prime} 2}(t)=v_{x_{0}^{\prime} 2}^{\mu \delta}(t), & & t \in[1 / 2,2], \\
\tilde{v}_{x_{0}^{\prime \prime 2} 2}(t)=v_{x_{0}^{\prime \prime 2}}^{\mu \delta}(t), & & t \in[1 / 2,2] .
\end{array}
$$

Траектории $\left(\tilde{x}_{x_{0}^{\prime}}(\cdot), \tilde{x}_{x_{0}^{\prime \prime}}(\cdot)\right)$, соответствующие управлениям (4.6), изображены на рис. 2 . Видно, что они наводятся внутрь терминального множества $\hat{M}$.

\section{Заключение}

В данной работе предложен метод решения расширенной задачи программного наведения для особых кластеров множества начальных состояний, решающий проблему применения алгоритма решения задачи пакетного наведения [10], которая эквивалентна задаче гарантированного позиционного наведения. Метод также позволяет получать более точные решения и для регулярных кластеров множества начальных состояний. В основе метода лежат переход к возмущенной расширенной задаче программного наведения и процедура аппроксимации, вообще говоря, нестрого выпуклого расширенного множества управления гладким выпуклым компактом [13] и сжатия целевого множества.

Авторы выражают благодарность С. Н. Аввакумову, Ю. Н. Киселеву и М. В. Орлову за полезные обсуждения результатов настоящей статьи.

\section{СПИСОК ЛИТЕРАТУРЫ}

1. Осипов Ю.С. Пакеты программ: подход к решению задач позиционного управления с неполной информацией // Успехи мат. наук. 2006. Т. 61, № 4. С. 25-76.

2. Кряжимский А.В., Осипов Ю.С. Идеализированные пакеты программ и задачи позиционного управления с неполной информацией // Тр. Ин-та математики и механики УрО РАН. 2009. Т. 15, № 3. С. $139-157$.

3. Красовский Н.Н., Субботин А.И. Позиционные дифференциальные игры. М.: Наука, 1974. $456 \mathrm{c}$. 
4. Кряжимский А.В., Осипов Ю.С. О разрешимости задач гарантирующего управления для частично наблюдаемых линейных динамических систем // Тр. МИАН. 2012. № 277. С. 152-167.

5. Кряжимский А.В., Стрелковский Н.В. Программный критерий разрешимости задачи позиционного наведения с неполной информацией. Линейные управляемые системы // Тр. Ин-та математики и механики УрО РАН. 2014. Т. 20, № 3. С. 132-147.

6. Стрелковский Н.В. Построение стратегии гарантированного позиционного наведения для линейной управляемой системы при неполной информации // Вестн. Моск. ун-та. Сер. 15: Вычисл. математика и кибернетика. 2015. № 3. С. 31-38.

7. Сурков П.Г. О задаче пакетного наведения с неполной информацией для линейной управляемой системы с запаздыванием // Проблемы динамического управления : сб. науч. тр. под ред. Ю. С. Осипова / фак. ВМК МГУ им. М. В. Ломоносова. № 7. М.: Изд. отдел фак-та ВМиК МГУ; MAKC Пресс, 2016. С. 94-108.

8. Максимов В.И., Сурков П. Г. О разрешимости задачи гарантированного пакетного наведения на систему целевых множеств. Вестн. Удмурт. ун-та. Математика. Механика. Компьют. науки. 2017. T. 27, № 3. С. 344-354.

9. Сурков П.Г. Задача пакетного наведения с неполной информацией при интегральном сигнале наблюдения // Сиб. электрон. мат. изв. 2018. Т. 15. С. 373-388.

10. Стрелковский Н.В., Орлов С.М. Алгоритм построения гарантирующего пакета программ в задаче управления при неполной информации // Вестн. Моск. ун-та. Сер. 15: Вычисл. математика и кибернетика. 2018. № 2. С. 20-31.

11. Strelkovskii N.V., Orlov S.M. A method for calculation of program package elements for singular clusters // Materials Internat. Conf. "Systems Analysis: Modeling and Control" in memory of Academician A. V. Kryazhimskii (Moscow, Russia, 2018). P. 97-99.

12. Гиндес В.Б. Об особом управлении в оптимальных системах // Изв. вузов. Математика. 1967. № 7 . С. $34-42$.

13. Аввакумов С.Н. Гладкая аппроксимация выпуклых компактов // Тр. Ин-та математики и механики УрО РАН. 1996. Т. 4. С. 184-200.

14. Киселёв Ю.Н., Аввакумов С.Н., Орлов М.В. Оптимальное управление. Линейная теория и приложения. М.: МАKC Пресс, 2007. 272 с.

15. Понтрягин Л.С. Линейные дифференциальные игры // Тр. МИАН. 1988. Т. 185. С. $200-207$.

16. Аввакумов С.Н., Киселёв Ю.Н., Орлов М.В. Методы решения задач оптимального управления на основе принципа максимума Понтрягина // Тр. МИАН. 1995. Т. 211. С. 3-31.

17. Аввакумов С.Н., Киселёв Ю.Н. Опорные функции некоторых специальных множеств, конструктивные процедуры сглаживания, геометрическая разность // Проблемы динамического управления: сб. науч. тр. под ред. Ю.С. Осипова, А.В.Кряжимского / фак. ВМК МГУ им. М. В. Ломоносова. № 1. М.: Изд. отдел фак-та ВМиК МГУ; МАКС Пресс, 2005. С. 24-110.

Поступила 23.11.2018

После доработки 27.12.2018

Принята к публикации 14.01.2019

Орлов Сергей Михайлович

канд. физ.-мат. наук

ассистент факультета вычислительной математики и кибернетики

МГУ им. М. В. Ломоносова, г. Москва

науч. сотрудник

Международный институт прикладного системного анализа, г. Лаксенбург

e-mail: sergey.orlov@cs.msu.ru

Стрелковский Никита Витальевич

канд. физ.-мат. наук, науч. сотрудник

Международный институт прикладного системного анализа, г. Лаксенбург

науч. сотрудник факультета вычислительной математики и кибернетики

МГУ им. М. В. Ломоносова, г. Москва

e-mail: strelkon@iiasa.ac.at 


\section{REFERENCES}

1. Osipov Yu.S. Control packages: an approach to solution of positional control problems with incomplete information. Russian Math. Surveys, 2006, vol. 61, no. 4, pp. 611-661. doi: 10.4213/rm1760 .

2. Kryazhimskiy A.V., Osipov, Y.S. Idealized program packages and problems of positional control with incomplete information. Proc. Steklov Inst. Math., 2010, vol. 268, suppl. 1, pp. 155-174. doi: $10.1134 /$ S0081543810050123 .

3. Krasovskii N.N., Subbotin A.I. Game-theoretical control problems. N Y: Springer, 1987. 517 p. This book is substantially revised version of the monograph Pozitsionnye differentsial'nye igry, Moscow, Nauka Publ., 1974, 456 p.

4. Kryazhimskiy A.V., Osipov Yu.S. On the solvability of problems of guaranteeing control for partially observable linear dynamical systems. Proc. Steklov Inst. Math., 2012, vol. 277, pp. 144-159. doi: 10.1134/S0081543812040104.

5. Kryazhimskii A.V., Strelkovskii N.V. An Open-loop criterion for the solvability of a closed-loop guidance problem with incomplete information: Linear control systems. Proc. Steklov Inst. Math., 2015, vol. 291, suppl. 1, pp. 113-127. doi: 10.1134/S0081543815090084.

6. Strelkovskii N.V. Constructing a strategy for the guaranteed positioning guidance of a linear controlled system with incomplete data. Moscow Univ. Comput. Math. Cybern., 2015, vol. 39, no. 3, pp. 126-134. doi: $10.3103 /$ S0278641915030085 .

7. Surkov P.G. The problem of package guidance with incomplete information for a linear control system with a delay. Comput Math Model, 2017, vol. 28, no. 4, pp. 504-516. doi: 10.1007/s10598-017-9377-y .

8. Maksimov V.I., Surkov P.G. On the solvability of the problem of guaranteed package guidance to a system of target sets. Vestn. Udmurtsk. Univ. Mat. Mekh. Komp. Nauki, 2017, vol. 27, no. 3, pp. 344-354 (in Russian). doi: $10.20537 / \mathrm{vm} 170305$.

9. Surkov P.G. The problem of package guidance under incomplete information and integral signal of observation. Sib. Elektron. Mat. Izv., 2018, vol. 15, pp. 373-388 (in Russian). doi: $10.17377 /$ semi.2018.15.034 .

10. Strelkovskii N.V., Orlov S.M. Algorithm for constructing a guaranteeing program package in a control problem with incomplete information. Moscow Univ. Comput. Math. Cybern., 2018, vol. 42, no. 2, pp. 69-79. doi: 10.3103/S0278641918020061.

11. Strelkovskii N.V., Orlov S.M. A method for calculation of program package elements for singular clusters. Proc. Int. Conf. "Systems Analysis: Modeling and Control" in memory of Academician A.V. Kryazhimskiy (Moscow, Russia, 2018), pp. 97-99. doi: 10.4213/proc20606 .

12. Gindes V.B. Singular control in optimal systems. Izv. Vyssh. Uchebn. Zaved. Mat., 1967, no. 7, pp. 34-42 (in Russian).

13. Avvakumov S.N. Smooth approximation of convex compact sets. Trudy Inst. Mat. i Mekh. UrO RAN, 1996, vol. 4, pp. 184-200 (in Russian).

14. Kiselev Yu.N., Avvakumov S.N., Orlov M.V. Optimal'noe upravlenie. Lineinaya teoriya i prilozheniya. [Optimal control. Linear theory and applications]. Moscow: MAKS Press, 2007, 272 p.

ISBN: 5-89407-288-3.

15. Pontryagin L.S. Linear differential games. Proc. Steklov Inst. Math., 1990, vol. 185, pp. 225-232.

16. Avvakumov S.N., Kiselev Yu.N., Orlov M.V., Methods of solving optimal control problems based on the Pontryagin maximum principle. Proc. Steklov Inst. Math., 1995, vol. 211, pp. 1-27.

17. Avvakumov S.N., Kiselev Yu.N. Support functions of some special sets, constructive smoothing procedures, and geometric difference. In: Osipov Yu.S. (ed.) et al., Problems in dynamic control. No. 1. Moscow: Mosk. Gos. Univ., Fak. Vych. Mat. Kib. Publ., 2005, pp. 24-110. ISBN: 5-89407-241-7.

Received November 23, 2018; Revised December 27, 2018; Accepted January 14, 2018

Funding Agency: This work was supported by the Russian Science Foundation (project no. 1411-00539).

Sergei Mikhailovich Orlov, Cand. Sci. (Phys.-Math.), Lomonosov Moscow State University, Moscow, 119991 Russia; Research Scholar, International Institute for Applied Systems Analysis, Laxenburg, 2361 Austria, e-mail: sergey.orlov@cs.msu.ru .

Nikita Vital'evich Strelkovskii. Cand. Sci. (Phys.-Math.), International Institute for Applied Systems Analysis, Laxenburg, 2361 Austria; Lomonosov Moscow State University, Moscow, 119991 Russia, e-mail: strelkon@iiasa.ac.at . 